\title{
Assignment of Reference 5'-end 16S rDNA Sequences and Species-Specific Sequence Polymorphisms Improves Species Identification of Nocardia
}

\author{
Fanrong Kong ${ }^{1}$, Sharon C.A. Chen ${ }^{1, *}$, Xiaoyou Chen ${ }^{1,2}$, Vitali Sintchenko ${ }^{1}$, Catriona Halliday ${ }^{1}$, \\ ${\text { Lin } \mathrm{Cai}^{3} \text {, Zhongsheng Tong }}^{1,4}$, Ok Cha Lee ${ }^{1}$, and Tania C. Sorrell ${ }^{1}$
}

\author{
${ }^{l}$ Centre for Infectious Diseases and Microbiology, The University of Sydney, Westmead Hospital, Westmead, New South \\ Wales, Australia \\ ${ }^{2}$ Department of Tuberculosis, Beijing Tuberculosis \& Thoracic Tumour Research Institute, Beijing, P. R. China \\ ${ }^{3}$ Department of Dermatology, Peking University People's Hospital, Beijing, P. R. China
}

${ }^{4}$ Research Laboratory for Infectious Skin Diseases, Department of Dermatology, Wuhan First Hospital, Wuhan, P. R. China

\begin{abstract}
S rDNA sequence analysis is the most accurate method for definitive species identification of nocardiae. However, conflicting results can be found due to sequence errors in gene databases. This study tested the feasibility of species identification of Nocardia by partial (5'-end 606-bp) 16S rDNA sequencing, based on sequence comparison with "reference" sequences of well-annotated strains. This new approach was evaluated using 96 American Type Culture Collection $(n=6)$, and clinical $(n=90)$ Nocardia isolates. Nucleotide sequence-based polymorphisms within species were indicative of "sequence types" for that species. Sequences were compared with those in the GenBank, Bioinformatics Bacteria Identification and Ribosomal Database Project databases. Compared with the reference sequence set, all 96 isolates were correctly identified using the criterion of $\geq 99 \%$ sequence similarity. Seventy-eight $(81.3 \%)$ were speciated by database comparison; alignment with reference sequences resolved the identity of $14(15 \%)$ isolates whose sequences yielded $100 \%$ similarity to sequences in GenBank under $>1$ species designation. Of 90 clinical isolates, the commonest species was Nocardia nova (33.3\%) followed by Nocardia cyriacigeorgica (26.7\%). Recently-described or uncommon species included Nocardia veterana (4.4\%), Nocarida bejingensis $(2.2 \%)$ and, Nocardia abscessus and Nocardia arthriditis (each $\mathrm{n}=1)$. Nocardia asteroides sensu stricto was rare $(\mathrm{n}=1)$. There were nine sequence types of $N$. nova, three of Nocardia brasiliensis with two each of $N$. cyriacigeorgica and Nocardia farcinica. Thirteen novel sequences were identified. Alignment of sequences with reference sequences facilitated species identification of Nocardia and allowed delineation of sequence types within species, suggesting that such a barcoding approach can be clinically useful for identification of bacteria.
\end{abstract}

Keywords: Nocardia spp., species identification, 16S rDNA, reference sequences, sequence polymorphisms.

\section{INTRODUCTION}

Nocardia species cause a range of infections including localised lung and skin infections, and disseminated disease. Speciation of clinical isolates is important to characterise associated disease manifestations, predict antimicrobial susceptibility and identify differences in epidemiology [1]. Since standard phenotypic identification methods are time-consuming and often imprecise [2, 3], nucleic acidamplification tools targeting conserved gene regions have been developed to facilitate accurate species determination.

Of these, 16S rDNA sequence analysis is the most frequently-used method for definitive species identification of nocardiae [2, 4-6]. Polymorphisms within the 65-kDa heat shock protein gene ( $h$ sp65) target are also reported to enable speciation $[7,8]$. These sequence-based identification

*Address correspondence to this author at the Centre for Infectious Diseases and Microbiology, Westmead Hospital, Darcy Road, Westmead, New South Wales, 2145, Australia; Fax: +61-2- 9891 5317;

E-mail: Sharon.chen@swahs.health.nsw.gov.au methods have led to substantial species re-assignment within the genus, especially among "Nocardia asteroides" isolates. Over 80 species have now been described of which at least 33 have been implicated in human disease (http://www. ncbi.nlm.nih.gov/Taxonomy/; http://www.bacterio.cict.fr/n/ nocardia.html; [2]).

Numerous Nocardia 16S rDNA sequences have thus been deposited in public sequence databases; however, a substantial proportion of sequences, for example in GenBank, represents misidentified isolates or contains significant errors [9, 10]. Imprecise species identification may also result from the presence of multiple, but different, copies of $16 \mathrm{~S}$ rDNA in certain Nocardia spp. such as Nocardia nova [11]. Further, sequence-based analyses are complicated by the lack of consensus regarding the degree of sequence similarity required for species definition of Nocardia [12].

To improve sequence-based species identification, there has been strong impetus to develop libraries of DNA sequences in order to designate, or link, standardised sequences, including nucleotide polymorphisms within these 
sequences, with a particular species; this process requires the establishment of such "reference sequences" or "DNA barcodes" for species identification and for the recognition of intraspecies sequence polymorphisms or "sequence types" $[13,14]$. Such an approach has not yet been applied to the identification of Nocardia. Since the few molecular analyses of Nocardia culture collections have reported significant species misidentification using phenotypic methods [5, 15], we re-examined the species identity of 96 Nocardia isolates in our collection using partial (5'-end 606-bp) 16S rDNA sequencing and the assignment of sequence types. The accuracy of three publicly-available gene databases for species identification was compared.

\section{MATERIALS AND METHODS}

\section{Nocardia Organisms}

Ninety-six Nocardia isolates were studied (supplementary Table S1; Table 1). These comprised six American Type Culture Collection (ATCC; Rockville, MD) strains (N. asteroides ATCC $19247^{\mathrm{T}}$, Nocardia farcinica ATCC 3308, N. farcinica ATCC $3318^{\mathrm{T}}$, N. nova ATCC $33726^{\mathrm{T}}$, Nocardia otitidiscaviarum ATCC $14629^{\mathrm{T}}$ and Nocardia paucivorans ATCC BAA-278 ${ }^{\mathrm{T}}$ ) and 90 clinical isolates (from the Clinical Mycology Laboratory, Centre for Infectious Diseases and Microbiology, Westmead Hospital, Sydney, Australia). Clinical isolates were cultured from separate patients from 1997-2005. All isolates were speciated using standard phenotypic methods and antibiotic susceptibility profiles [3] and, by $16 \mathrm{~S}$ rDNA sequencing.
Isolates were cultured aerobically in brain heart infusion broth (Amyl Media, Dandenong, Australia) for 3-15 days at $37^{\circ} \mathrm{C}$.

\section{DNA Extraction}

Cells from 2 ml-brain heart infusion broth cultures of Nocardia in late logarithmic phase were harvested by centrifugation at $14,000 \mathrm{Xg}$ for $10 \mathrm{~min}$. The supernatant was removed and the pellet suspended in $150 \mu \mathrm{l}$ of digestion buffer (10 mM Tris-HCl [pH 8.0], 0.45\% Triton X-100 and $0.45 \%$ Tween 20). Bacterial suspensions were then heated for $10 \mathrm{~min}$ at $100^{\circ} \mathrm{C}$ to lyse the cells, followed by cooling at $-20^{\circ} \mathrm{C}$ for $1 \mathrm{~h}$. Cell lysates were centrifuged at $14,000 \mathrm{X} g$ for $5 \mathrm{~min}$ to pellet the cell debris. Supernatants containing DNA were diluted in $350 \mu \mathrm{TE}$ buffer $(5 \mathrm{mM}$ Tris $\mathrm{HCl}, 0.5$ mM EDTA) and centrifuged for 2 min to remove cell debris. DNA was quantitated using a spectrophotometer and stored at $-20^{\circ} \mathrm{C}$ until required.

\section{PCR Amplification and Sequencing of the 16S rDNA}

The 5'-end 606-bp fragment of the 16S rDNA gene was amplified using the universal bacterial primers $16 \mathrm{~S}-27 \mathrm{f0}$ (5' to 3': 1 TTA GAG TTT TGA TCM TGG CTC 21) and 16S-907r (5' to 3': 986 CCG TCA ATT CMT TRA GTT T 877) [16]. Each PCR reaction contained $5 \mu \mathrm{l}$ template DNA, $0.25 \mu \mathrm{l}(50 \mathrm{pmol} / \mu \mathrm{l})$ each of forward primer and reverse primer, $1.25 \mu \mathrm{l}$ dNTPs $(2.5 \mathrm{mM}$ of each dNTP: Roche Diagnostics, Mannheim, Germany), $2.5 \mu 1$ 10x PCR buffer (Qiagen, Donacaster, Victoria), 0.1 $\mu$ l HotStar Taq polymerase $(5 \mathrm{U} / \mu \mathrm{l})$ and water to a $25 \mu \mathrm{l}$ final volume. Amplifica-

Table 1. Species Distribution of 96 Nocardia Isolates by Sequence-Based Alignment of 606-bp 16S rDNA Fragments with Reference Sequences and BLASTn Against Database Sequences

\begin{tabular}{|c|c|c|c|c|c|}
\hline N. abscessus & $1 / 0$ & $3^{2}$ & $100 / 15$ & $100 / 20$ & $100 / 13$ \\
\hline N. aobensis & $1 / 0$ & 1 & $99.8 / 4$ & $99.8 / 4$ & $98.8 / 4$ \\
\hline N. beijingensis & $2 / 0$ & 1 & $100 / 13$ & $100 / 18$ & $98.1 / 13$ \\
\hline N. brasiliensis & $3 / 3$ & 1 & $100 / 2$ & $100 / 4$ & $100 / 2$ \\
\hline N. cyriacigeorgica & $24 / 0$ & 1 & $99.8-100 / 35$ & $99.8-100 / 21$ & $99.1-100 / 15$ \\
\hline N. farcinica & $13 / 10$ & $2^{4}$ & $99.8-100 / 14$ & $100 / 12$ & $100 / 8$ \\
\hline N. transvalensis & $1 / 0$ & 1 & $99.7 / 3$ & $99.6 / 5$ & $98.8 / 2$ \\
\hline$N$. veterana & $4 / 0$ & 1 & $100 / 5$ & $100 / 8$ & $100 / 4$ \\
\hline N. vinacea & $1 / 0$ & 1 & $100 / 3$ & $100 / 3$ & $100 / 2$ \\
\hline
\end{tabular}

${ }^{1}$ Refers to the number strains in the database with a sequence similarity of $\geq 99 \%$ to that of the query sequence.

${ }^{2}$ Refers to $100 \%$ sequence similarity with $N$. asteroides, $N$. asiatica and $N$. abscessus sequences.

${ }^{3} 99.6 \%$ sequence similarity to a single $N$. bejingensis sequence (GenBank accession no. AY756543) but $<99 \%$ sequence similarity to other $N$. bejingensis strains. Since the sequence demonstrated $99.0 \%$ similarity to the reference $N$. arthriditis sequence, it was assigned as such.

${ }^{4}$ Refers to $100 \%$ sequence similarity with $N$. farcinica sequences as well as with sequence of $N$. otitidiscavarium strain DSM $43242{ }^{\mathrm{T}}$ (GenBank accession no. X80611). 
tion was performed in a Mastercycler gradient thermocycler (Eppendorf; Netheler-Hinz GmbH, Germany). The cycling conditions were: $95^{\circ} \mathrm{C}$ for $15 \mathrm{~min}$ followed by 35 cycles of $94^{\circ} \mathrm{C}$ for $30 \mathrm{~s}, 55^{\circ} \mathrm{C}$ for $30 \mathrm{~s}, 72^{\circ} \mathrm{C}$ for $90 \mathrm{~s}$ with a final extension step at $72^{\circ} \mathrm{C}$ for $10 \mathrm{~min}$.

PCR products were purified (PCR Product Presequencing Kit; USB Corporation, Cleveland, $\mathrm{OH}$ ) and sequenced using the BigDye Terminator version 3.1 cycle sequencing kit (ABI PRISM 3100 genetic analyser; Applied Biosystems, Foster City, CA) and the primer $16 \mathrm{~S}-27 \mathrm{f}$ (5' to 3': 3 AGA GTT TTG ATC MTG GCT CAA G 23) [16]. Each sequence was manually aligned and analysed to ensure high quality sequence data. Where the sequence of an isolate differed from the GenBank "reference" sequence for that species (see Results and Table 2), [7, 17, 18] or for novel sequences, additional primers (16S-27f0 and 16S-907r) were used to confirm the result of the sequence.

\section{S rDNA Sequence Analysis}

For each isolate, the amplified 5'-606 bp 16S rDNA fragment was examined using the BioManager facility (ANGIS, Sydney; http://biomanager.angis.org.au/). Consensus sequences were constructed from alignments of sequence data using ClustalW [19] after careful examination of each electrophoregram trace representation of data. Sequence data were queried against archived sequences in the GenBank (BLASTn 2.2.10; http://www.ncbi.nlm.nih.gov), Bioinformatics Bacteria Identification (BIBI) version 0.2 (http:// umr5558-sud-str1.univ-lyon1.fr/lebibi/lebibi.cgi; [20]) and Ribosomal Database Project-II (RDP-II) version 9.54 (http://rdp.cme.msu.edu/index.jsp; [21]) databases.

A list of the closest sequence matches was generated from the database comparisons with pair-wise distance scores indicating the percent similarity between the unknown (query) sequence and database sequences. Only consensus sequences with a minimum length of 606-bp were analysed. A percent similarity (or identity) score of $\geq 99 \%[4,5]$ was used as the criterion to classify an isolate to species level whilst a 97 to $98.9 \%$ similarity score identified an isolate as belonging to the genus Nocardia but to a different species $[9,10]$.

\section{Nucleotide Sequence Accession Number}

Thirty-four 606 bp partial 16S rDNA sequences including 13 novel Nocardia sequences generated in the study were deposited in GenBank with the following accession numbers (also see Table 3): FJ172101 through to FJ172134.

\section{RESULTS}

\section{Establishment of a Reference Set of 16S rDNA Sequences}

For this study, the 606-bp 16S rDNA sequences of 43 well-characterised isolates representing 43 taxonomicallyauthenticated Nocardia species (http://www.bacterio.cict.fr/ n/nocardia.html) were chosen as the reference sequences for that species; these isolates were previously-characterised by both $16 \mathrm{~S}$ rRNA and $h s p 65$ gene analyses [7, 8]. The sequences of two additional isolates, Nocardia elegans DSM $44890^{\mathrm{T}}$ and Nocardia aobensis IFM 0137 [17, 18], were included in the reference sequence set (Table 2). Examination of the $N$. asteroides ATCC 14759 sequence
(N. asteroides type VI, GenBank accession no. DQ223862) found it to be identical to that of Nocardia cyriacigeorgica DSM $44484^{\mathrm{T}}$ (GenBank accession no. AY756550). The sequence of Nocardia beijingensis JCM $10666^{\mathrm{T}}$ (GenBank accession no. AY756543) reported in one study [6, 7] was $98.5 \%$ similar to that of this same strain (GenBank accession no. DQ659901) described in a separate study [17]. We assigned the sequence corresponding to accession no. DQ659901 as the reference sequence for $N$. beijingensis since this yielded the higher similarity $(100 \%)$ to multiple $N$. beijingensis sequences in the GenBank, BIBI and RDP-II databases.

This collection of partial $16 \mathrm{~S}$ rDNA sequences representing 45 Nocardia species formed the basis for re-evaluating the species identity of the study isolates and was important in the validation of the sequence analyses. Strains fulfilling the criterion for a species ( $\geq 99 \%$ sequence similarity) but which demonstrated sequence polymorphisms compared to the reference sequence for that species were considered as separate sequence types for the species.

\section{S rDNA Sequence-Based Identification of Nocardia Isolates}

The details of 96 Nocardia isolates identified by phenotypic methods and partial $16 \mathrm{~S}$ rDNA sequencing are given in supplementary Table $\mathbf{S 1}$. The species distribution of isolates, as determined by sequence comparison with the reference sequence set, and with sequences in the GenBank, BIBI and RDP-II databases, is shown in Table 1.

\section{(a) Identification Based on Comparison with Reference 606-bp 16S rDNA Sequences}

Following alignment of sequence data with the reference sequence set, partial $16 \mathrm{~S}$ rDNA sequencing provided species identification for all 96 isolates using a criterion of $\geq 99 \%$ sequence similarity for species definition; $83(86.5 \%)$ isolates were identified if a criterion of $100 \%$ sequence similarity was used.

Of 90 clinical isolates, phenotypic methods correctly identified $42(46.7 \%)$ strains to species level (Table 1; supplementary Table S1). Thirty-four isolates were assigned a phenotypic identification of $N$. asteroides $N$. asteroides complex based on their drug susceptibility pattern but $16 \mathrm{~S}$ rDNA sequencing recognised them as a number of distinct species e.g. N. cyriacigeorgica, $N$. farcinica and $N$. paucivorans among others (Supplementary Table S1). Molecular and phenotypic species identification methods were concordant for $80 \%$ (8 of 10 ), $75 \%$ (three of four), $90 \%$ (27 of 30) and 100\% (all of three) of N. farcinica, $N$. otitidiscavarium, $N$. nova and Nocardia brasiliensis clinical isolates, respectively. However, none of the six $N$. paucivorans isolates were correctly identified by phenotypic methods. All six ATCC strains were assigned by $16 \mathrm{~S}$ rDNA sequencing to their respective species (Supplementary Table S1).

Discrepant results for isolates are summarised in Supplementary Table S1 (see also Table 1). In particular, only one of 35 phenotypic " $N$. asteroides/N. asteroides complex" isolates (other than strain ATCC 19247") had a sequence identical to sequences of $N$. asteroides sensu 
Table 2. Reference 5' end 606-bp 16S rDNA Sequences of 45 Nocardia Species ${ }^{1}$ used in the Present Study

\begin{tabular}{|c|c|c|}
\hline Strain no. ${ }^{1,2}$ & Species Identification & GenBank Accession no. ${ }^{3}$ \\
\hline DSM $44432^{\mathrm{T}}$ & N. abscessus & AY544980 \\
\hline IFM 0137 & N. aobensis & AB126875 (bp positions 40-645) \\
\hline DSM $44729^{\mathrm{T}}$ & N. araoensis & AY903623 \\
\hline DSM $44668^{\mathrm{T}}$ & N. asiatica & AY903617 \\
\hline ATCC $19247^{\mathrm{T}}$ & N. asteroids & AY756541 \\
\hline ATCC 49872 & N. asteroides type IV & AY756542 \\
\hline JCM $10666^{\mathrm{T}}$ & N. beijingensis & DQ6599014 (bp positions 14-619) \\
\hline DSM $43397^{\mathrm{T}}$ & N. carnea & AY756546 \\
\hline DSM $44546^{\mathrm{T}}$ & N. cerradoensis & AY756547 \\
\hline ATCC $700418^{\mathrm{T}}$ & N. crassostreae & AY756548 \\
\hline DSM $44490^{\mathrm{T}}$ & N. cummidelens & AY756549 \\
\hline DSM $44484^{\mathrm{T}}$ & N. cyriacigeorgica & AY756550 \\
\hline ATCC 14759 & N. asteroides type VI & DQ223862 \\
\hline DSM $44890^{\mathrm{T}}$ & N. elegans & DQ659905 (bp positions 1-603) \\
\hline $\operatorname{DSM} 43665^{\mathrm{T}}$ & N. farcinica & AY756551 \\
\hline $\operatorname{DSM} 44717^{\mathrm{T}}$ & N. neocaledoniensis & AY903614 \\
\hline DSM $44670^{\mathrm{T}}$ & N. niigatensis & AY903615 \\
\hline CIP $104777^{\mathrm{T}}$ & N. nova & AY756555 \\
\hline ATCC $14629^{\mathrm{T}}$ & N. otitidiscaviarum & AY756556 \\
\hline DSM $44386^{\mathrm{T}}$ & N. paucivorans & AY756557 \\
\hline DSM $44730^{\mathrm{T}}$ & N. pneumonia & AY903622 \\
\hline DSM $44290^{\mathrm{T}}$ & N.pseudobrasiliensis & AY756558 \\
\hline DSM $43406^{\mathrm{T}}$ & N. pseudovaccinii & AY756559 \\
\hline DSM $44599^{\mathrm{T}}$ & N. puris & AY903618 \\
\hline $\mathrm{JCM} 4826^{\mathrm{T}}$ & N. salmonicida & AY756560 \\
\hline $\operatorname{DSM} 44129^{\mathrm{T}}$ & N. seriolae & AY756561 \\
\hline $\operatorname{DSM} 44733^{\mathrm{T}}$ & N. shimofusensis & AY903621 \\
\hline DSM $44488^{\mathrm{T}}$ & N. soli & AY756562 \\
\hline DSM $44704^{\mathrm{T}}$ & N. tenerifensis & AY903613 \\
\hline DSM $44765^{\mathrm{T}}$ & N. testacea & AY903612 \\
\hline DSM $43405^{\mathrm{T}}$ & N. transvalensis & AY756563 \\
\hline
\end{tabular}


Table 2. contd...

\begin{tabular}{|c|c|c|}
\hline Strain no., & Species Identification & GenBank Accession no. $^{\mathbf{3}}$ \\
\hline \hline $\mathrm{JCM} 3224^{\mathrm{T}}$ & N. uniformis & AY756564 \\
\hline ATCC $11092^{\mathrm{T}}$ & N. vaccinii & AY756565 \\
\hline $\mathrm{DSM} 44445^{\mathrm{T}}$ & N. veteran & AY756566 \\
\hline $\mathrm{JCM} 10988^{\mathrm{T}}$ & N. vinacea & AY756567 \\
\hline DSM $44669^{\mathrm{T}}$ & N. yamanashiensis & AY903616 \\
\hline
\end{tabular}

${ }^{1}$ All species designations were cross-checked against two websites - http://www.ncbi.nlm.nih.gov/Taxonomy/ and http://www.bacterio.cict.fr/n/nocardia.html. Abbreviations: ATCC, American Type Culture Collection; CIP, Collection Institut Pasteur, France; DSM, Deutsche Sammling von Mikroorganismen und Zellkulturen GmbH, Germany; JCM, Japan Collection of Microorganisms, Wako-Shi, Japan. Adapted from [7, 17, 18].

${ }^{2} \mathrm{~T}$ refers to the previously-designated current type strains for the particular species.

${ }^{3}$ Unless otherwise specified refers to first $1-606$ bp of the 16 S rDNA sequence from the 5 'end.

${ }^{4}$ Different sequence results obtained for $N$. beijingensis JCM $10666^{\mathrm{T}}$ in two separate studies. The sequence with GenBank accession no. DQ659901 is chosen as the reference sequence.

${ }^{5}$ The sequence of $N$. cyriacigeorgica strain DSM $44490^{\mathrm{T}}$ (GenBank accession no. AY756550) is identical to the sequence of N. asteroides ATCC 14759 (accession no. DQ223862)

${ }^{6}$ The sequence is based on a 603-bp 16S rDNA fragment.

stricto (represented by N. asteroides ATCC $19247^{\mathrm{T}}$ ); 22 clinical isolates had sequences with $100 \%$ similarity to the reference $N$. cyriacigeorgica sequence. The remaining strains were $N$. paucivorans $(\mathrm{n}=3), N$. nova $(\mathrm{n}=2), N$. beijingensis $(\mathrm{n}=2)$, Nocardia abscessus $(\mathrm{n}=1)$, Nocardia arthritidis $(\mathrm{n}=1), N$. farcinica $(\mathrm{n}=1)$ and Nocardia veterana $(\mathrm{n}=1)$. Other discrepant results included two $N$. farcinica (phenotypic identification) isolates yielding sequences with $100 \%$ similarity to the reference $N$. cyriacigeorgica sequence and a $N$. brasiliensis strain with $100 \%$ sequence similarity to N. otitidiscavarium.

Eleven isolates (supplementary Table S1) identified as "Nocardia spp." by phenotypic methods were identified as $N$. paucivorans $(\mathrm{n}=3), N$. farcinica, $N$. veterana $($ each $\mathrm{n}=2)$ and $N$. aobensis, N. nova, Nocardia transvalensis and Nocardia vinacea (each $\mathrm{n}=1)$.

\section{b) Species Identification Based on BLASTn Alignments}

Isolates were also identified to species level by comparison with database sequences with the following exceptions: firstly, $N$. farcinica could not be definitively speciated; all 13 strains (100\% sequence similarity to the reference $N$. farcinica sequence) were identified as either $N$. farcinica or $N$. otitidiscavarium (Table 1). The three databases contained a sequence corresponding to $N$. otitidiscavarium strain DSM $43242^{\mathrm{T}}$ (GenBank accession no. X80611). This sequence was indistinguishable from $N$. farcinica sequences but only had $94.7 \%$ similarity to the reference sequence of N. otitidiscavarium (GenBank accession no. AY756556). Secondly, a phenotypic $N$. asteroides isolate was identified as $N$. asteroides/N. abscessus/Nocardia asiatica (Table 1). Since its sequence was identical to the reference sequence of $N$. abscessus, it was assigned as such. The reference $16 \mathrm{~S}$ rDNA sequences of $N$. abscessus and $N$. asiatica (Table 2) differ only by a single nucleotide polymorphism (SNP) at position 527 ("G" for $N$. abscessus but " $\mathrm{C}$ " for $N$. asiatica). Finally, an isolate (strain 01-320-2714; supplementary Table S1) was identified as $N$. beijingensis by database comparisons $(99.6 \%$ sequence similarity to a single $N$. bejingensis sequence; GenBank accession no. AY756543). However, as it yielded $99 \%$ sequence similarity (5-bp difference) to the reference sequence of $N$. arthriditis, it was assigned as N. arthriditis (Table 1).

\section{Comparison of Species Identification Using GenBank, BIBI and RDP-II Databases}

For all isolates, the same species identification result was obtained by comparison of their sequences with those in the GenBank, BIBI and RDP-II databases (Table 1). The distribution of percent similarity scores according to database is shown in Fig (1). Using the criterion of $\geq 99 \%$ sequence similarity for species designation, all 96 isolates were identified by the GenBank and BIBI databases and 91 (96.7\%), by the RDP-II system. The length of sequences employed for sequence alignment in the BIBI, RDP-II and GenBank databases ranged from 512-548 bp, 572-590 bp and $\geq 606 \mathrm{bp}$, respectively. Perfect matches (100\% similarity) were observed for $77 \%, 79 \%$ and $93 \%$ of sequence alignments against the GenBank, BIBI and RDP-II databases, respectively (Fig. 1).

\section{Species Distribution of Clinical Isolates}

Fourteen Nocardia species were identified amongst 90 isolates, the most common being $N$. nova (30 isolates; 33\%) followed by $N$. cyriacigeorgica $(\mathrm{n}=24 ; 27 \%), N$. farcinica $(\mathrm{n}=11 ; 12 \%)$ and $N$. paucivorans $(\mathrm{n}=6 ; 7 \%)$. There were four isolates each of $N$. otitidiscaviarum and $N$. veterana, three strains of $N$. brasiliensis, two of $N$. beijingensis and one each of $N$. asteroides sensu stricto, $N$. transvalensis, $N$. vinacea, $N$. aobensis, $N$. arthritidis and N. abscessus.

\section{Intra-Species Variation and Sequence Types of Clinical Isolates}

Partial 16S rRNA sequences of $N$. veterana, N. abscessus, $N$. beijingensis and $N$. vinacea isolates were identical to the reference sequence for that species, thus there was a single sequence type. Intraspecies sequence heterogeneity was evident in the remaining 10 species, and varied with species (Table 3). The largest number of sequence types, including that identical to the reference sequence, was noted for $N$. nova (nine sequence types) followed by $N$. brasiliensis (three sequence types). N. cyriacigeorgica, N. farcinica, $N$. asteroides sensu stricto and $N$. otitidiscavarium exhibited two sequence types. The sequences of three $N$. cyriacigeorgica isolates (indistinguishable from one another) differed from the reference $N$. cyriacigeorgica sequence by a SNP at position 576 (substitution of "A" for "G", see Table 3). 
Table 3. Partial (5'-end 606-bp) 16S rDNA Sequence Polymorphisms in 10 Nocardia Species

\begin{tabular}{|c|c|c|c|c|}
\hline $\begin{array}{l}\text { Strain } \\
\text { Identification no. }\end{array}$ & $\begin{array}{l}\text { Identification } \\
\text { Based on Comparison } \\
\text { with Reference Sequence }\end{array}$ & $\begin{array}{l}\text { \% Similarity } \\
\text { to Reference } \\
\text { Sequence }\end{array}$ & $\begin{array}{l}\text { Site of Nucleotide Polymorphisms } \\
\text { (bp Position: Reference Sequence } \rightarrow \text { Isolate } \\
\text { Sequence) }\end{array}$ & $\begin{array}{l}100 \% \text { Similarity to Another GenBank Sequences } \\
\text { Rather Than Reference Sequences (13 Novel } \\
\text { Sequence GenBank Accession no.) }\end{array}$ \\
\hline 04-303-0576 & N. aobensis & 99.67 & $137 \mathrm{G} \rightarrow \mathrm{A}$ & novel sequence $^{1}(\mathrm{FJ} 172101)$ \\
\hline $01-320-2714$ & N. arthritidis & 99.0 & $\begin{array}{l}132 \mathrm{C} \rightarrow \mathrm{T}, 240 \mathrm{G} \rightarrow \mathrm{A}, 251 \mathrm{C} \rightarrow \mathrm{T}, \\
341 \mathrm{C} \rightarrow \mathrm{G}, 566 \mathrm{~A} \rightarrow \mathrm{G}, 588 \mathrm{G} \rightarrow \mathrm{T}\end{array}$ & novel sequence ${ }^{1}(\mathrm{FJ} 172102)$ \\
\hline $02-071-3627$ & N. asteroides & 99.0 & $\begin{array}{l}133-135 \mathrm{TTC} \rightarrow \mathrm{ACA}, \\
148-150 \mathrm{GAG} \rightarrow \mathrm{TGT}\end{array}$ & novel sequence ${ }^{1}(\mathrm{FJ} 172103)$ \\
\hline 00-194-3516 & N. brasiliensis & 99.84 & $203 \mathrm{~T} \rightarrow \mathrm{C}$ & Z36935 \\
\hline $03-273-2825$ & N. brasiliensis & 99.67 & $203 \mathrm{~T} \rightarrow \mathrm{C}, 328 \mathrm{G} \rightarrow \mathrm{A}$ & AY245543 \\
\hline $99-167-2395$ & N. brasiliensis & 99.67 & $203 \mathrm{~T} \rightarrow \mathrm{C}, 328 \mathrm{G} \rightarrow \mathrm{A}$ & AY245543 \\
\hline $00-159-1584$ & N. cyriacigeorgica & 99.84 & $576 \mathrm{G} \rightarrow \mathrm{A}$ & \multirow[t]{3}{*}{ novel sequence ${ }^{1}(\mathrm{FJ} 172112)$} \\
\hline 04-181-3939 & N. cyriacigeorgica & 99.84 & $576 \mathrm{G} \rightarrow \mathrm{A}$ & \\
\hline $05-111-2308$ & N. cyriacigeorgica & 99.84 & $576 \mathrm{G} \rightarrow \mathrm{A}$ & \\
\hline 01-109-2248 & N. farcinica & 99.84 & $67 \mathrm{~A} \rightarrow \mathrm{A} / \mathrm{G}^{2}$ & novel sequence $^{1}(\mathrm{FJ} 172117)$ \\
\hline $05-053-4454$ & N. nova & 99.84 & $86 \mathrm{C} \rightarrow \mathrm{C} / \mathrm{T}^{2}$ & novel sequence ${ }^{1}(\mathrm{FJ} 172126)$ \\
\hline $02-352-3316$ & N. nova & 99.84 & $136 \mathrm{G} \rightarrow \mathrm{A} / \mathrm{G}^{2}$ & novel sequence $^{1}(\mathrm{FJ} 172120)$ \\
\hline $00-130-2170$ & N. nova & 99.84 & $260 \mathrm{~A} \rightarrow \mathrm{A} / \mathrm{G}^{2}$ & novel sequence ${ }^{1}(\mathrm{FJ} 172121)$ \\
\hline 04-110-3287 & N. nova & 99.67 & $86 \mathrm{C} \rightarrow \mathrm{C} / \mathrm{T}^{2}, 136 \mathrm{G} \rightarrow \mathrm{A} / \mathrm{G}^{2}$ & novel sequence ${ }^{1}(\mathrm{FJ} 172125)$ \\
\hline 00-056-3529 & N. nova & 99.67 & $86 \mathrm{C} \rightarrow \mathrm{T}, 136 \mathrm{G} \rightarrow \mathrm{A}$ & \multirow[t]{4}{*}{ AF430030 } \\
\hline 01-067-1349 & N. nova & 99.67 & $86 \mathrm{C} \rightarrow \mathrm{T}, 136 \mathrm{G} \rightarrow \mathrm{A}$ & \\
\hline 01-097-0996 & N. nova & 99.67 & $86 \mathrm{C} \rightarrow \mathrm{T}, 136 \mathrm{G} \rightarrow \mathrm{A}$ & \\
\hline $02-199-2723$ & N. nova & 99.67 & $86 \mathrm{C} \rightarrow \mathrm{T}, 136 \mathrm{G} \rightarrow \mathrm{A}$ & \\
\hline $00-025-0538$ & N. nova & 99.67 & $135 \mathrm{G} \rightarrow \mathrm{T}, 148 \mathrm{~T} \rightarrow \mathrm{G}$ & \multirow[t]{3}{*}{ AF430032 } \\
\hline $00-314-1789$ & N. nova & 99.67 & $135 \mathrm{G} \rightarrow \mathrm{T}, 148 \mathrm{~T} \rightarrow \mathrm{G}$ & \\
\hline 04-150-0614 & N. nova & 99.67 & $135 \mathrm{G} \rightarrow \mathrm{T}, 148 \mathrm{~T} \rightarrow \mathrm{G}$ & \\
\hline 01-066-1903 & N. nova & 99.51 & $86 \mathrm{C} \rightarrow \mathrm{T}, 136 \mathrm{G} \rightarrow \mathrm{A}, 578 \mathrm{~T} \rightarrow \mathrm{G}$ & novel sequence ${ }^{1}(\mathrm{FJ} 172122)$ \\
\hline 02-021-0419 & N. nova & 99.51 & $135 \mathrm{G} \rightarrow \mathrm{T}, 148 \mathrm{~T} \rightarrow \mathrm{G}, 328 \mathrm{~A} \rightarrow \mathrm{G}$ & novel sequence ${ }^{1}$ (FJ172119) \\
\hline 01-114-2816 & N. otitidiscaviarum & 99.85 & $133 \mathrm{~A} \rightarrow \mathrm{G}$ & novel sequence ${ }^{1}$ (FJ172127) \\
\hline ATCC BAQ-278 & N. paucivorans & 99.67 & $33 \mathrm{C}$-ins $^{3}, 37 \mathrm{G}$-ins ${ }^{3}$ & \multirow[t]{7}{*}{ AF179865 } \\
\hline 03-141-3073 & N. paucivorans & 99.67 & $33 \mathrm{C}$-ins $^{3}, 37 \mathrm{G}$-ins ${ }^{3}$ & \\
\hline 03-185-3304 & N. paucivorans & 99.67 & 33 C-ins ${ }^{3}, 37$ G-ins ${ }^{3}$ & \\
\hline $03-240-2758$ & N. paucivorans & 99.67 & $33 \mathrm{C}^{-i n s^{3}}, 37 \mathrm{G}-$ ins $^{3}$ & \\
\hline $05-200-1797$ & N. paucivorans & 99.67 & $33 \mathrm{C}^{-i n s^{3},}, 37 \mathrm{G}-$ ins $^{3}$ & \\
\hline $97-114-0609$ & N. paucivorans & 99.67 & $33 \mathrm{C}^{-i n s^{3}}, 37 \mathrm{G}-$ ins $^{3}$ & \\
\hline $97-16298$ & N. paucivorans & 99.67 & $33 \mathrm{C}^{-i n s^{3},}, 37 \mathrm{G}-$ ins $^{3}$ & \\
\hline 03-310-2776 & N. transvalensis & 99.67 & $402,403 \mathrm{AG} \rightarrow \mathrm{CA}$ & novel sequence ${ }^{1}(\mathrm{FJ} 172131)$ \\
\hline
\end{tabular}

${ }^{1}$ Sequences of isolates without a match with GenBank sequences are novel sequences.

${ }^{2}$ At the specified bp position, both nucleotides were present due to different multiple copies of the 16S rRNA gene.

${ }^{3}$ Refers to insertion of the specified base.

There was only one sequence type for $N$. aobensis, $N$. transvalensis, $N$. arthritidis and $N$. paucivorans but the sequences of these isolates all demonstrated SNPs when compared to the reference sequence for the species (Table 3 ). Thirteen novel Nocardia sequences were identified for 15 isolates (eight species).

\section{DISCUSSION}

Molecular-based identification of Nocardia spp. remains a challenge due to the increasing recognition of new species and changes in taxonomy [22]. Sequence analysis of the $16 \mathrm{~S}$ rDNA is the current gold standard for identification of Nocardia spp. However few studies have explored the 


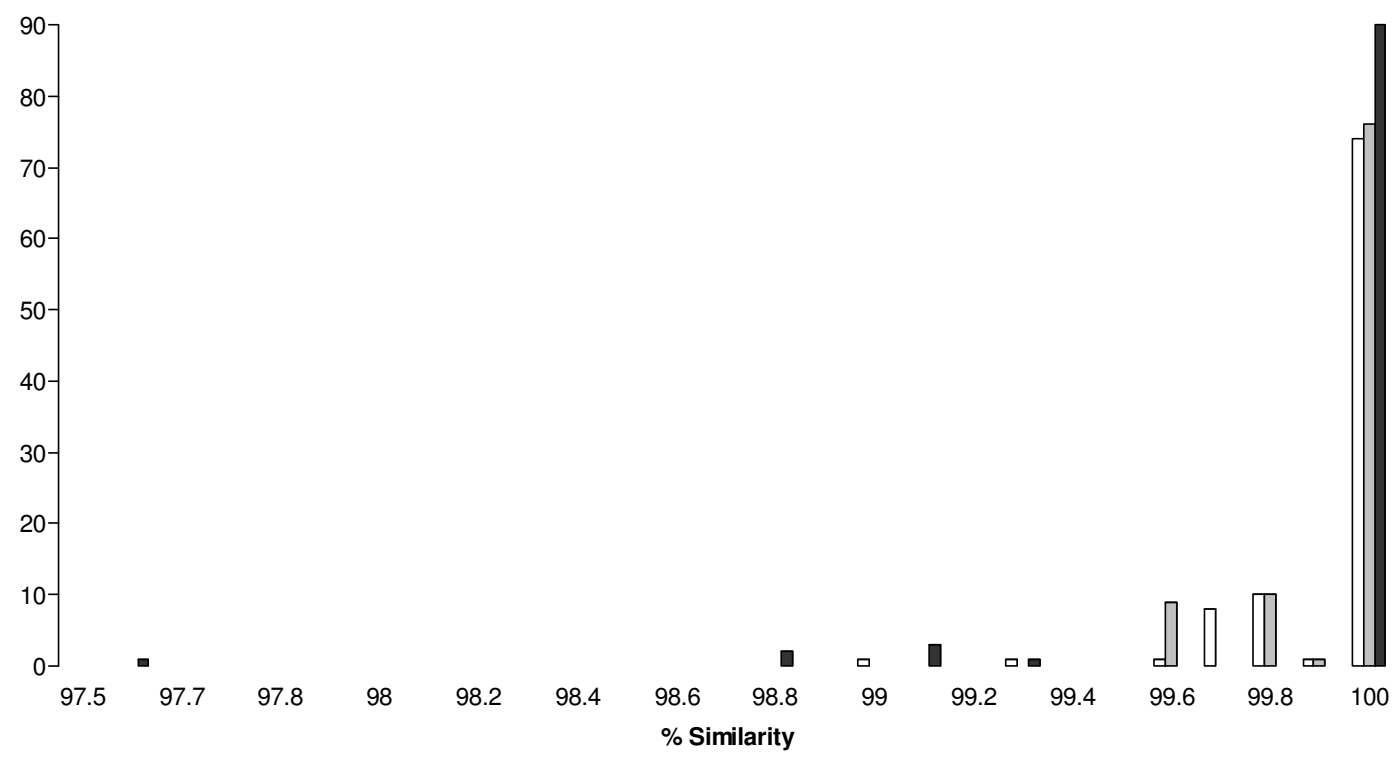

$\square$ GenBank $\square$ BIBI $\square$ RDP-II

Fig. (1). Distribution of similarity scores for partial 16S rDNA sequence-based identification of Nocardia isolates by the GenBank, BIBI and RDP-II databases.

validity of sequence-based identification approaches using collections of phenotypically-characterised clinical isolates [9]. The present study proposes, and has tested the utility of a set of reliable reference Nocardia 16S rDNA sequences, derived from authoritatively-identified organisms [7, 17], as sequence standards for species identification. Further, by identifying and assigning sequence types to represent sequence polymorphisms within a species, the results have identified that such a "barcoding" approach can improve systematic and accurate species identification. DNA barcoding has been designed to provide rapid accurate species identification by using short, standardised gene regions (in this case, the 5'-end $16 \mathrm{~S}$ rDNA region) as internal species tags [14]. Although it has been found to be effective in speciating eukaryote organisms and some parasites $[13,14,16]$, there are few data on its application in the identification of bacterial pathogens.

As such, based on comparison with the reference sequence set, partial $16 \mathrm{~S}$ rDNA sequencing provided clear species identification of all 96 isolates. In particular, the reference sequence set was useful in the speciation of 11 isolates identified only to genus level by phenotypic methods and assisted in resolving the identity of $14(15 \%)$ clinically relevant isolates whose sequences aligned with $100 \%$ similarity to sequences assigned to more than one species in the GenBank, BIBI and RDP-II databases (Table 1). Without the resource of this sequence set, sequencing was unable to assign precise species identification to $13 \mathrm{~N}$. farcinica isolates (Table 1). It is most likely that the $N$. otitisdiscavarium DSM $43242^{\mathrm{T}}$ sequence (GenBank accession no. X80611) with $100 \%$ identity to N. farcinica sequences represents a misidentification, underscoring the importance of adequate stewardship of database sequences. Of note, the reference sequence of $N$. abscessus and $N$. asiatica (GenBank accession nos. AY544980 and AY903617, respectively) differ only by a SNP - this low interspecies heterogeneity likely explains the inability to resolve species identifi- cation for $N$. abscessus after alignment with databases sequences (Table 3). N. abscessus (previously N. asteroides antimicrobial susceptibility type I) represents $\approx 20 \%$ of isolates of the former $N$. asteroides complex; accurate species identification is important as MICs of imipenem, which is commonly used to treat nocardiosis, are high for this species [2]. There are few descriptions of $N$. asiatica as a pathogen.

Species identification of Nocardia by gene sequence analysis is heavily reliant on the entries in the gene repositories being queried $[10,23]$. As noted in the present study, inappropriate and /or obsolete sequence entries are important potential limitations. Further, species identification based on data derived from a single or small numbers of strains representing a species must be interpreted with caution (see also Table 1). As such, the submission of carefullyannotated new sequences is critical to maintaining the accuracy of current gene repositories. Since the BIBI and RDP-II systems contain a larger proportion of shorter $16 \mathrm{~S}$ rDNA sequences (512-548 bp, 572-590 bp, respectively vs. $\geq 606$ bp in GenBank), this may have resulted in an artificially inflated number of perfect sequence matches (Fig. 1). The validity of comparisons with sequences of different lengths for species identification requires further study.

The approach undertaken in this study has further allowed us to distinguish between closely-related Nocardia species, to identify newly described or uncommon species and to determine the species distribution of clinical Nocardia isolates received in our laboratory. Elsewhere and in Australia, most human infections historically have been attributed to $N$. asteriodes sensu stricto antimicrobial susceptibility class types I and VI, N. nova, $N$. brasiliensis and $N$. farcinica $[1,24]$. Our results reconfirm that this generally remains the case. Given the prevalence of $N$. cyriacigeorgica (previously N. asteroides type VI as a pathogen (this study; [25]), adop- 
tion of protocols by microbiology laboratories for its identification is important. Apart from distinguishing N. abscessus from $N$. asiatica (see above), partial 16S rDNA sequencing was able to differentiate between other closely-related species including $N$. veterana and $N$. nova sensu stricto (sequence similarity of $98.1 \% ;[6,26,27])$. Classified within the $N$. nova complex, $N$. veterana is an emerging pathogen capable of causing serious infection [27]. Of note, the re-assignment of all but one clinical "N. asteroides" strains to other taxonomic groups questions the validity of $N$. asteroides as a separate species.

Importantly, the results identified significant intraspecies sequence polymorphisms within the $16 \mathrm{~S}$ rDNA for many (10 of 14) Nocardia species or for seven of nine species represented by more than one strain (Table 3 ), and that such nucleotide heterogeneity differed according to species, being most evident for $N$. nova (nine sequence types). Although there was no genetic heterogeneity amongst isolates of, for example, $N$. farcinica, the sequences of the isolates differed from the reference sequence for this species. Thus, if constructing a DNA template or "identification barcode" for identifying Australian N. paucivorans isolates, 16S rDNA position 33 should incorporate an extra $\mathrm{C}$ and at position 37, an extra $G$ in relation to the reference sequence (Table $\mathbf{3}$ ). In the USA, three "genetic types" of $N$. cyriacigeorgica with SNPs at positions 448, 1427 and 1480 [25] have been reported; we identified a SNP at position 576 but not at position 448. Thus, comparison of sequence types of isolates from different countries to identify potential clinical and epidemiological associations may be warranted. As noted for other bacteria, substitutions of as little as 1-2 bp may correlate with unique Nocardia phenotypes and clinical significance $[5,28]$. Therefore, documentation of sequence polymorphisms within species is relevant to delimiting species or highlighting genetically-distinct groups with levels of sequence divergence that are either suggestive or exclusive of species status.

Finally, 13 novel sequences from eight Nocardia species were identified and some of them may merit description as new species (Table 3). Species designation, however, is confounded by the lack of a consensus criterion for species definition based on percent similarity scores; isolates of distinct species of Nocardia have been reported to exhibit as much as $99.8 \%$ sequence similarity [12].

\section{CONCLUSION}

Partial 16S rDNA sequencing is a viable alternative to full-length sequencing for species identification of Nocardia in a diagnostic laboratory. The present approach encompassed comparison of the sequence of interest with a library of reference sequences and the assigning of sequence types to represent sequence polymorphisms within species, based on sequence similarity to the reference sequence for that species; unambiguous species identification was obtained for all study isolates. As affirmed in the present study, errors in sequence entries remain important potential limitations of public gene repositories $[6,10,23]$. The results of the study suggest that a barcoding approach $[29,30]$ can assist with species identification of clinically relevant Nocardia. Further studies are warranted to explore its wider application in improving species differentiation and unravelling sequence data for phylogenetically-unresolved groups of bacteria.

\section{SUPPLEMENTARY MATERIAL}

Supplementary material is available on the publishers Web site along with the published article.

\section{ACKNOWLEDGMENTS}

We thank Ms. Maryann Pincevic for her assistance in performing the 16S rDNA sequencing and Ms. Ping Zhu for help with the Figure preparation.

\section{REFERENCES}

[1] Saubolle MA, Sussland D. Nocardiosis: review of clinical and laboratory experience. J Clin Microbiol 2003; 41: 4497-501.

[2] Brown-Elliott BA, Brown JM, Conville PS, Wallace RJ, Jr. Clinical and laboratory features of the Nocardia spp. based on current molecular taxonomy. Clin Microbiol Rev 2006; 19: 259-82.

[3] McNeil MM, Brown JM. The medically important aerobic Actinomycetes: epidemiology and microbiology. Clin Microbiol Rev 1994; 7: 357-417.

[4] Cloud JL, Conville PS, Croft A, Harmsen D, Witebsky FG, Carroll KC. Evaluation of partial $16 \mathrm{~S}$ ribosomal DNA sequencing for identification of Nocardia species by using the MicroSeq 500 system with an expanded database. J Clin Microbiol 2004; 42: 57884.

[5] Roth A, Andrees S, Kroppenstedt RM, Harmsen D, Mauch H. Phylogeny of the genus Nocardia based on reassessed 16S rRNA gene sequences reveals underspeciation and division of strains classified as Nocardia asteroides into three established species and two unnamed taxons. J Clin Microbiol 2003; 41: 851-6.

[6] Mellmann A, Cloud JL, Andrees S, et al. Evaluation of RIDOM, MicroSeq, and Genbank services in the molecular identification of Nocardia species. Int J Med Microbiol 2003; 293: 359-70.

[7] Rodriguez-Nava V, Couble A, Devulder G, Flandrois JP, Boiron P, Laurent F. Use of PCR-restriction enzyme pattern analysis and sequencing database for hsp65 gene-based identification of Nocardia species. J Clin Microbiol 2006; 44: 536-46.

[8] Steingrube VA, Brown BA, Gibson JL, et al. DNA amplification and restriction endonuclease analysis for differentiation of 12 species and taxa of Nocardia, including recognition of four new taxa within the Nocardia asteroides complex. J Clin Microbiol 1995; 33: 3096-101.

[9] Clarridge JE, III. Impact of 16S rRNA gene sequence analysis for identification of bacteria on clinical microbiology and infectious diseases. Clin Microbiol Rev 2004; 17: 840-62.

[10] Janda JM, Abbott SL. 16S rRNA gene sequencing for bacterial identification in the diagnostic laboratory: pluses, perils, and pitfalls. J Clin Microbiol 2007; 45: 2761-4.

[11] Conville PS, Witebsky FG. Analysis of multiple differing copies of the $16 \mathrm{~S}$ rRNA gene in five clinical isolates and three type strains of Nocardia species and implications for species assignment. J Clin Microbiol 2007; 45: 1146-51.

[12] Conville PS, Witebsky FG. Multiple copies of the 16S rRNA gene in Nocardia nova isolates and implications for sequence-based identification procedures. J Clin Microbiol 2005; 43: 2881-5.

[13] Savolainen V, Cowan RS, Vogler AP, Roderick GK, Lane R. Towards writing the encyclopedia of life: an introduction to DNA barcoding. Philos Trans R Soc Lond B Biol Sci 2005; 360: 180511.

[14] Hebert PD, Gregory TR. The promise of DNA barcoding for taxonomy. Syst Biol 2005; 54: 852-9.

[15] Wauters G, Avesani V, Charlier J, Janssens M, Vaneechoutte M, Delmee M. Distribution of Nocardia species in clinical samples and their routine rapid identification in the laboratory. $\mathrm{J}$ Clin Microbiol 2005; 43: 2624-8.

[16] Becker K, Harmsen D, Mellmann A, et al. Development and evaluation of a quality-controlled ribosomal sequence database for $16 \mathrm{~S}$ ribosomal DNA-based identification of Staphylococcus species. J Clin Microbiol 2004; 42: 4988-95. 
[17] Conville PS, Zelazny AM, Witebsky FG. Analysis of secAl gene sequences for identification of Nocardia species. J Clin Microbiol 2006; 44: 2760-6.

[18] Kageyama A, Suzuki S, Yazawa K, Nishimura K, Kroppenstedt RM, Mikami Y. Nocardia aobensis sp. nov., isolated from patients in Japan. Microbiol Immunol 2004; 48: 817-22.

[19] Thompson JD, Higgins DG, Gibson TJ. CLUSTAL W: improving the sensitivity of progressive multiple sequence alignment through sequence weighting, position-specific gap penalties and weight matrix choice. Nucleic Acids Res 1994; 22: 4673-80.

[20] Devulder G, Perriere G, Baty F, Flandrois JP. BIBI, a bioinformatics bacterial identification tool. J Clin Microbiol 2003; 41: 1785-7.

[21] Cole JR, Chai B, Farris RJ, et al. The ribosomal database project (RDP-II): introducing myRDP space and quality controlled public data. Nucleic Acids Res 2007; 35: D169-D72.

[22] Patel JB, Wallace RJ, Jr., Brown-Elliott BA, et al. Sequence-based identification of aerobic Actinomycetes. J Clin Microbiol 2004; 42: 2530-40.

[23] Petti CA. Detection and identification of microorganisms by gene amplification and sequencing. Clin Infect Dis 2007; 44: 1108-14.
[24] Georghiou PR, Blacklock ZM. Infection with Nocardia species in Queensland. A review of 102 clinical isolates. Med J Aust 1992; 156: 692-7.

[25] Schlaberg R, Huard RC, Della-Latta P. Nocardia cyriacigeorgica is an emerging pathogen in the United States. J Clin Microbiol 2007; 46: $265-73$.

[26] Gurtler V, Smith R, Mayall BC, Potter-Reinemann G, Stackebrandt E, Kroppenstedt RM. Nocardia veterana sp. nov., isolated from human bronchial lavage. Int J Syst Evol Microbiol 2001; 51: 933-6.

[27] Pottumarthy S, Limaye AP, Prentice JL, Houze YB, Swanzy SR, Cookson BT. Nocardia veterana, a new emerging pathogen. J Clin Microbiol 2003; 41: 1705-9.

[28] Tortoli E. Impact of genotypic studies on mycobacterial taxonomy: the new mycobacteria of the 1990s. Clin Microbiol Rev 2003; 16 : 319-54.

[29] Blaxter M, Mann J, Chapman T, et al. Defining operational taxonomic units using DNA barcode data. Philos Trans R Soc Lond B Biol Sci 2005; 360: 1935-43.

[30] Ferri G, Alu M, Corradini B, Licata M, Beduschi G. Species identification through DNA "barcodes". Genet Test Mol Biomarkers 2009; [Epub ahead of print] PMID: 19405876. 